\title{
VARIACIÓN EN LA ABUNDANCIA DE ÁRBOLES MADERABLES POR EFECTO DE BORDE EN UN BOSQUE TROPICAL SUBCADUCIFOLIO
}

\section{VARIATION IN THE ABUNDANCE OF TIMBER TREES BY EDGE EFFECT IN A TROPICAL SUBDECIDUOUS FOREST}

\author{
Víctor Abraham Vargas-Vázquez ${ }^{1}$, Crystian Sadiel Venegas-Barrera ${ }^{2}$, Arturo Mora-Olivo ${ }^{1, *}$, José Guadalupe \\ Martínez-Ávalos ${ }^{1}$, Eduardo Alanís-Rodríguez ${ }^{3}$, Edilia de la Rosa-Manzano ${ }^{1}$ \\ ${ }^{1}$ Universidad Autónoma de Tamaulipas, Instituto de Ecología Aplicada, Ciudad Victoria, Tamaulipas, México \\ 2 Tecnológico Nacional de México / Instituto Tecnológico de Ciudad Victoria, Departamento de Posgrado e Investigación, Ciudad \\ Victoria, Tamaulipas, México \\ ${ }^{3}$ Universidad Autónoma de Nuevo León, Facultad de Ciencias Forestales, Linares, Nuevo León, México
}

"Autor de correspondencia: amorao@uat.edu.mx

\section{Resumen}

Antecedentes: El efecto de borde afecta diferencialmente a las especies en sus estadios de desarrollo. El presente estudio analizó las condiciones ambientales asociadas a la abundancia por estadio de desarrollo de cuatro especies de árboles maderables en el borde de un bosque tropical subcaducifolio.

Hipótesis: Los bordes presentan mayor incidencia lumínica y temperatura, condiciones favorables para las plántulas, por lo que se espera que en el borde se presente la mayor abundancia de plántulas con respecto al interior del bosque.

Especies en estudio: Bursera simaruba (L.) Sarg., Cedrela odorata L., Guazuma ulmifolia Lam., Lysiloma divaricatum (Jacq.) J.F. Macbr. Sitio de estudio y fechas: Reserva de la Biosfera "El Cielo" (Tamaulipas), México. Enero-diciembre 2016.

Métodos: La abundancia por estadios de desarrollo y las condiciones ambientales fueron cuantificadas en el gradiente. Dichas variables se correlacionaron, además se contrastaron los requerimientos entre estadios y se asociaron con los ambientes identificados.

Resultados: Guazuma ulmifolia mostró una respuesta negativa al efecto de borde, mientras que Cedrela odorata respondió positivamente. Los requerimientos ambientales difirieron entre los primeros estadios de desarrollo y los adultos. La abundancia de las plántulas se asoció a condiciones de mayor incidencia lumínica.

Conclusiones: Los requerimientos ambientales afectan diferencialmente a cada estadio de desarrollo. La abundancia de plántulas se incrementa en condiciones de mayor incidencia lumínica, pero no en el resto de los estadios, excepto en C. odorata. La pérdida de cobertura y la consecuente formación de bordes pueden llevar a una reducción en la abundancia de estas especies, con implicaciones económicas.

Palabras clave: Árboles maderables, bosque tropical subcaducifolio, condiciones ambientales, efecto de borde, estadios de desarrollo.

\begin{abstract}
Background: The edge effect differentially affects the species in their life stages. We analyzed the environmental conditions associated with the abundance by life stage of four species of timber trees on the edge of a subdeciduous tropical forest.

Hypothesis: The edges have higher light incidence and temperature, favorable conditions for the seedlings, so it is expected that the edge will have more abundance of seedlings with respect to the forest interior.

Species under study: Bursera simaruba (L.) Sarg., Cedrela odorata L., Guazuma ulmifolia Lam., Lysiloma divaricatum (Jacq.) J.F. Macbr. Study site and dates: Reserva de la Biosfera "El Cielo" (Tamaulipas), Mexico. January-December 2016.

Methods: The abundance by life stages and environmental conditions were quantified within the gradient. These variables were correlated, in addition the requirements between stages were contrasted and they were associated with the identified environments.

Results: Guazuma ulmifolia showed a negative response to the edge effect, while Cedrela odorata responded positively. The environmental requirements differed between the first life stages and adults. The abundance of the seedlings was associated to conditions of higher light incidence.

Conclusions: Environmental requirements differentially affect each life stage. The abundance of seedlings increases in conditions of higher light incidence, but not in the rest of the stages, except in $C$. odorata. The loss of cover and the consequent formation of borders can lead to a reduction in the abundance of these species, with economic implications.
\end{abstract}

Key words: Edge effect, environmental conditions, life stages, timber species, tropical subdeciduous forest.

This is an open access article distributed under the terms of the Creative Commons Attribution License CCBY-NC (4.0) international. https://creativecommons.org/licences/by-nc/4.0/ 
Los árboles maderables de los bosques tropicales representan una fuente de materia prima para la fabricación de herramientas y viviendas en las comunidades rurales (HernándezSandoval et al. 1991, Fuentes-Salinas et al. 2008, MorenoCasasola \& Paradowska 2009). La abundancia de los árboles maderables se encuentra determinada por su historia de vida, las condiciones específicas de humedad, temperatura e incidencia lumínica (Silva-Magnago et al. 2015). La perturbación incrementa la apertura del dosel, el área foliar efectiva, la radiación directa sobre el dosel, el índice de transmisión de luz, la humedad y temperatura. Tales variables tienen un efecto diferente en el desarrollo de los árboles, las cuales se relacionan con la germinación (Figueroa \& Lusk 2001), el reclutamiento (Montgomery \& Chazdon 2002, Lusk et al. 2006), la fotosíntesis (Poorter \& Arets 2003, Sapijanskas et al. 2014) y el crecimiento (Rüger et al. 2011). La temperatura ambiental y la humedad relativa afectan los procesos fisiológicos, como el desarrollo o el crecimiento secundario (Way \& Oren 2010, Cheesman \& Winter 2013), lo cual repercute en la abundancia de cada especie (Nava-Cruz et al. 2007). Sin embargo, las características de temperatura, humedad e incidencia lumínica se ven modificadas dentro de las áreas conservadas que se encuentran adyacentes a sitios modificados por el hombre, debido al efecto de borde (Murcia 1995, López-Barrera 2004, Ewers \& Banks-Leite 2013). El efecto de borde se define como la formación de un gradiente ambiental al interior de un área conservada con vegetación nativa adyacente a áreas modificadas antrópicamente, generado por la interacción entre ambos ambientes (López-Barrera 2004). Dicho gradiente ambiental influye directamente en la germinación, establecimiento y mortandad de las especies de árboles maderables, lo que causa variaciones en la abundancia de dichas especies (Nava-Cruz et al. 2007, Sugiyama \& Peterson 2013, Silva-Magnago et al. 2015, Suzán-Azpiri et al. 2017). Por lo tanto, el efecto de borde es un factor que determina la estructura de categorías de tamaños a lo largo de este gradiente (Williams-Linera \& Lorea 2009). Por ejemplo, durante el estadio de plántula del alejo (Caesalpinia platyloba) y el jocote (Spondias purpurea), la mortandad se incrementa proporcionalmente a la disminución de la humedad en las zonas cercanas al borde (Nava-Cruz et al. 2007). Mientras que, en el estadio adulto, la temperatura ambiental se relaciona positivamente con el crecimiento del tallo y la floración, los cuales son características claves para el manejo de especies maderables (Way \& Oren 2010, Athayde \& Morellato 2014). Asimismo, el éxito del manejo de las especies de árboles maderables depende de su abundancia y desarrollo, por lo cual, su disminución puede tener repercusiones económicas negativas. Por ello, el manejo y la conservación de las especies de árboles maderables tropicales requieren del conocimiento de las respuestas de la abundancia al efecto de borde dependiendo el estadio de desarrollo en que se encuentren.

En México, la mayoría de los estudios que analizan el efecto de borde se han realizado en bosques templados (López-Barrera \& Newton 2005). Sin embargo, especies tropicales como la chaca (Bursera simaruba), el cedro rojo (Cedrela odorata), el aquiche (Guazuma ulmifolia) y el rajador (Lysiloma divaricatum), son pioneras demandantes de luz que aprovechan los claros en el bosque para iniciar rápidamente el proceso de colonización, y así regenerar los sitios abandonados (Teketay \& Granström 1997, Pearson et al. 2002, 2003). Estas especies representan el $69 \%$ del volumen maderable total en México (INEGI 2007). Por ello, el conocimiento del efecto de borde a lo largo de los estadios de desarrollo es indispensable en el manejo y conservación de estas especies (Williams-Linera \& Lorea 2009, Sugiyama \& Peterson 2013), principalmente en los ecosistemas susceptibles a la pérdida de cobertura vegetal (Nava-Sosa et al. 2010).

Los bosques tropicales son uno de los ecosistemas que presentan la mayor pérdida de cobertura vegetal (Challenger et al. 2009, Thier \& Wesenberg 2016), como sucede en la Reserva de la Biosfera "El Cielo" (RBEC) (Steinberg et al. 2014). En esta reserva, los bosques tropicales subcaducifolios fueron explotados en diferentes periodos para fines agrícolas y forestales (Sánchez-Ramos et al. 2005), siendo este tipo de vegetación en donde se distribuyen las principales especies maderables utilizadas en la región (Hernández-Sandoval et al. 1991, Valiente-Banuet et al. 1995). Las características de los bosques tropicales de la RBEC, así como de las especies que inciden en ella, confieren un escenario propicio para analizar la relación entre las condiciones ambientales y la abundancia de los diferentes estadios de desarrollo de las plantas (plántulas, juveniles, adultos juveniles y adultos maduros) ante el efecto de borde.

El presente trabajo analizó la relación de la temperatura del aire, la humedad relativa y la incidencia lumínica en la abundancia de los diferentes estadios de desarrollo de cuatro especies maderables de un bosque tropical subcaducifolio, bajo condiciones de borde en la Reserva de la Biosfera "El Cielo". Se comparó la asociación de las características físicoambientales y la abundancia entre los estadios de desarrollo, así como su distribución a lo largo del gradiente ambiental. El estudio propuso que la abundancia de las plántulas y juveniles se encontrarían relacionadas positivamente con las condiciones ambientales del interior. Los resultados obtenidos pueden ser la base para realizar estudios futuros relacionados con el manejo y conservación de especies forestales de esta área natural protegida.

\section{Materiales y métodos}

Area de estudio. El sitio de estudio se localiza en el Ejido Nuevo Pensar del Campesino del municipio de Gómez Farías, al sur del estado de Tamaulipas, Noreste de México (INEGI 2006) (Figura 1). La localidad se encuentra dentro de los límites de la Reserva de la Biosfera "El Cielo", un área natural protegida de jurisdicción estatal (CONABIO 2015). El clima es cálido subhúmedo (CONABIO 1998) y el suelo dominante es litosol (INIFAP \& CONABIO 1995). La elevación es de 285 m s.n.m., con una pendiente menor a $10^{\circ}$ (INEGI 2013). El sitio de muestreo es un fragmento de bosque tropical subcaducifolio, no perturbado al menos en los últimos 40 años, el cual es adyacente a un área de cultivo de nopal con 20 años de abandono. El gradiente ambiental 


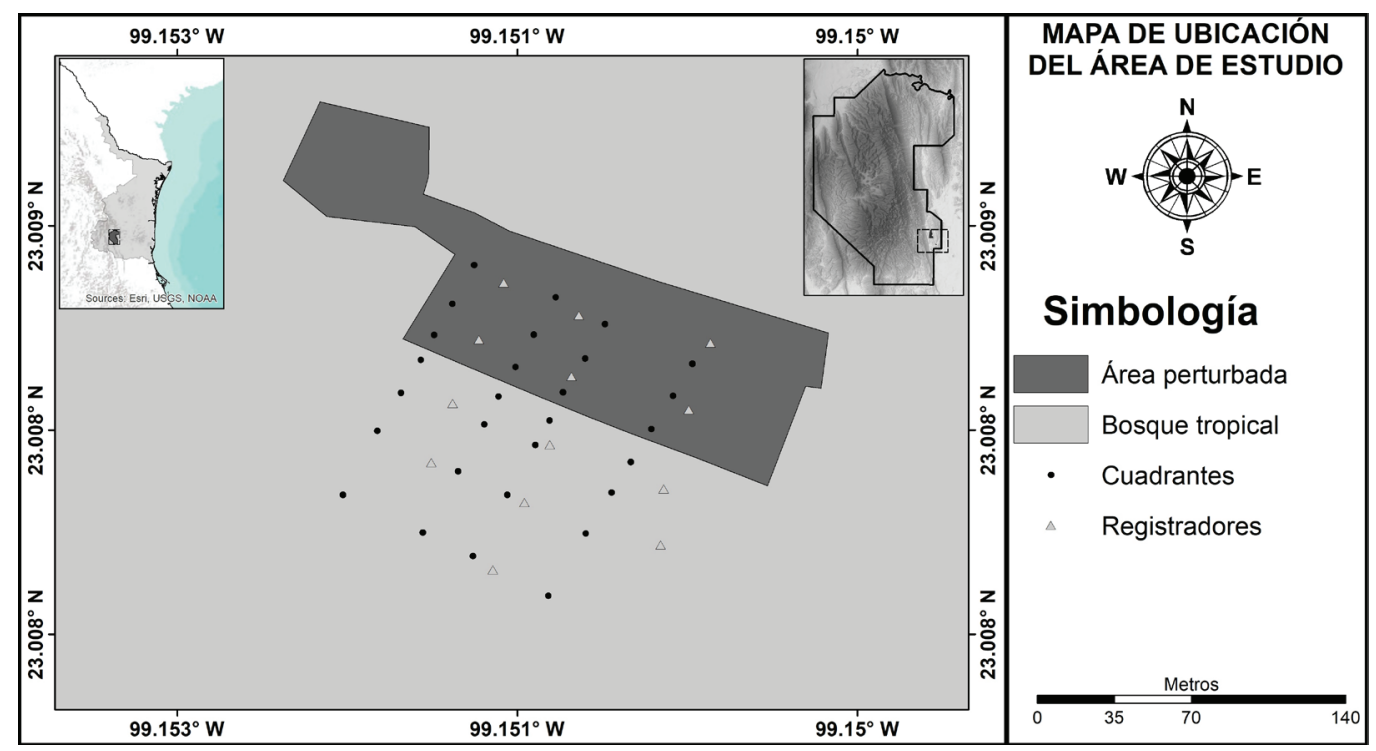

Figura 1. Mapa de ubicación del área de estudio.

generado entre el área no perturbada y el área abandonada por 20 años es considerado como un borde suave (sensu Ries et al. 2004), lo cual permite analizar el efecto de borde tanto en las plántulas como en los adultos.

Selección del sitio de muestreo. El sitio de evaluación fue elegido en función de la persistencia o ausencia de cobertura vegetal entre los años de 1986 y 2014, lo cual fue obtenido de la clasificación supervisada de escenas satelitales Landsat. Las escenas satelitales Landsat son imágenes con una resolución espacial de $30 \mathrm{~m}$, y en $170 \times 185 \mathrm{~km}$ registran el espectro electromagnético reflejado por lo objetos en la región de la luz visible (tres bandas), el infrarrojo cercano (dos bandas) y medio (una banda). Los objetos que se encuentran en la superficie de la Tierra, y desentendiendo de las propiedades físicas, reflejan diferencialmente el espectro electromagnético y esta propiedad permite identificar superficies con características similares. Las escenas fueron obtenidas de las plataformas Global Visualization (GLOVIS, http://www.glovis.usgs.gov) y Global Land Cover Facility (GLCF, http://www.glcf.umiacs.umd.edu). Las escenas fueron segmentadas para agrupar a los pixeles con características similares, con el objetivo de facilitar la selección de campo de entrenamiento, para posteriormente realizar una clasificación supervisada dura y obtener los tipos de cobertura de cada escena (Barrera-Salazar et al. 2015). La clasificación se realizó mediante el algoritmo de máxima verosimilitud, el cual permite clasificar los pixeles en las categorías con las cuales tenga mayor probabilidad de pertenecer (Lillesand et al. 2014, Barrera-Salazar et al. 2015). Las escenas clasificadas se utilizaron para identificar un área que fue cultivada $\mathrm{y}$ abandonada hace 20 años, contigua a vegetación no perturbada desde hace 40 años (Harper \& Macdonald 2002, Harper et al. 2005). Los procesamientos de las escenas satelitales se realizaron utilizando el programa IDRISI Selva 17.0 (Clark
Labs 2014), mientras que la selección de los sitios se realizó con el programa ArcGis 10.2.2 (ESRI 2014).

Especies de estudio. Previo al estudio, se realizó un muestreo piloto para seleccionar a las especies maderables que se analizarían, tomando como referencia a aquellas con mayor abundancia. El estudio piloto consistió en registrar el número de individuos de cada especie en un transecto de $200 \mathrm{~m}$ perpendicular al límite entre ambos ambientes. Las especies de árboles maderables más abundantes fueron Bursera simaruba (L.) Sarg. (chaca), Cedrela odorata L. (cedro rojo), Guazuma ulmifolia Lam. (aquiche) y Lysiloma divaricatum (Jacq.) Benth. (rajador). Bursera simaruba es un árbol mediano que alcanza los $20 \mathrm{~m}$ de altura con un diámetro a la altura del pecho (dap) de 40 a $80 \mathrm{~cm}$ (hasta $1 \mathrm{~m}$ ), se distribuye desde el sur de Florida y las islas Bahamas a través de las Antillas hasta Sur América; prospera en laderas abiertas y pedregosas, también en laderas de cerros a menudo formados por rocas metamórficas y terrenos planos. Se utiliza como un árbol ornamental, setos vivientes, entre otros. Cedrela odora$t a$, es un árbol con una altura de 12-30 m y un dap de 60-150 $\mathrm{cm}$. La especie ocurre naturalmente desde México hasta Sur América; se encuentra con frecuencia en pastizales y tierras de cultivo como árbol aislado; es una especie maderable muy importante debido a su alta calidad de la madera (Salas 1993). Guazuma ulmifolia, es un árbol mediano o arbusto de 2 a $15 \mathrm{~m}$ de altura con un dap de 30 a $40 \mathrm{~cm}$; es ampliamente distribuido desde México hasta América del Sur. Es considerada como una especie pionera tardía según sus habilidades competitivas (Herrera et al. 1997). Se reporta su uso como forraje, leña y sombra para el ganado. Lysiloma divaricatum es un árbol que alcanza de 3 a $5 \mathrm{~m}$ de altura, se distribuye desde México hasta Costa Rica. Las cuatro especies son una fuente importante de madera en la región, son usadas para la construcción de viviendas, la elaboración de herramientas y 
posterío (Hernández-Sandoval et al. 1991). Además, la calidad de su madera es óptima para uso comercial e industrial (Bárcenas-Pazos 1995, Fuentes-Salinas et al. 2008, MorenoCasasola \& Paradowska 2009).

Diseño de muestreo. Las evaluaciones para registrar a las especies fueron realizadas en cuatro transectos de $140 \mathrm{~m}$ perpendiculares al borde, separados entre sí por $20 \mathrm{~m}$. En los transectos se delimitaron siete cuadrantes de $10 \times 10 \mathrm{~m}$, de los cuales cuatro se encontraron a los 10, 20, 40 y $80 \mathrm{~m}$ al interior del área conservada y tres en el área perturbada a los 10, 20 y $40 \mathrm{~m}$ del borde (Figura 2). El área conservada se caracterizó por la presencia de especies de lento crecimiento o susceptibles a la perturbación como Enterolobium cyclocarpum (Jacq.) Griseb., Beaucarnea recurvata Lem. y Bromelia pinguin L.; así como la ausencia de especies indicadoras de perturbación como Acacia farnesiana (L.) Willd. (Valiente-Banuet et al. 1995). Mientras que el área perturbada se diferenció porque la cobertura vegetal fue eliminada para creación de áreas de cultivo de nopal, las cuales fueron abandonadas a partir de 1984.

Muestreo. Cada uno de los individuos registrados se asignó a una categoría de estadio de desarrollo, en función de sus características morfológicas, que incluyó plántulas, juveniles, adultos juveniles y adultos maduros (Alvarez-Buylla \& Martinez-Ramos 1992). Las plántulas incluyeron a los individuos recientemente establecidos (provistos solo de cotiledones), así como individuos con una altura menor a $30 \mathrm{~cm}$ (catáfilos presentes, nomófilos ausentes). El estadio juvenil consideró a los individuos con nomófilos desarrollados (cotiledones y catáfilos ausentes), eje principal sin ramificaciones, ejes laterales desarrollados, tallo con un diámetro menor a $5 \mathrm{~cm}$ y una altura menor a $1.5 \mathrm{~m}$. Los individuos categorizados como adultos juveniles se caracterizaron por presentar ejes laterales de tercer orden desarrollados, eje principal sin ramificaciones, ausencia de estructuras reproductivas, tallo con un diámetro menor a $10 \mathrm{~cm}$ y una altura menor a $2.5 \mathrm{~m}$. El estadio adulto maduro incluyó a individuos que presentaban ramificaciones, estructuras reproductivas presentes, tallo con un diámetro mayor a $10 \mathrm{~cm}$ y una altura mayor a $2.5 \mathrm{~m}$. La abundancia de cada especie, por categoría de desarrollo, fue registrada en cada cuadrante.

Caracterización ambiental de los sitios. La caracterización ambiental se realizó considerando la radiación lumínica, la humedad relativa y la temperatura ambiental. La radiación lumínica, se analizó a partir de imágenes hemisféricas tomadas con una cámara digital Canon EOS 5D Mark III, con un objetivo zoom ultra angular de ojo de pez USM (EF 8-15

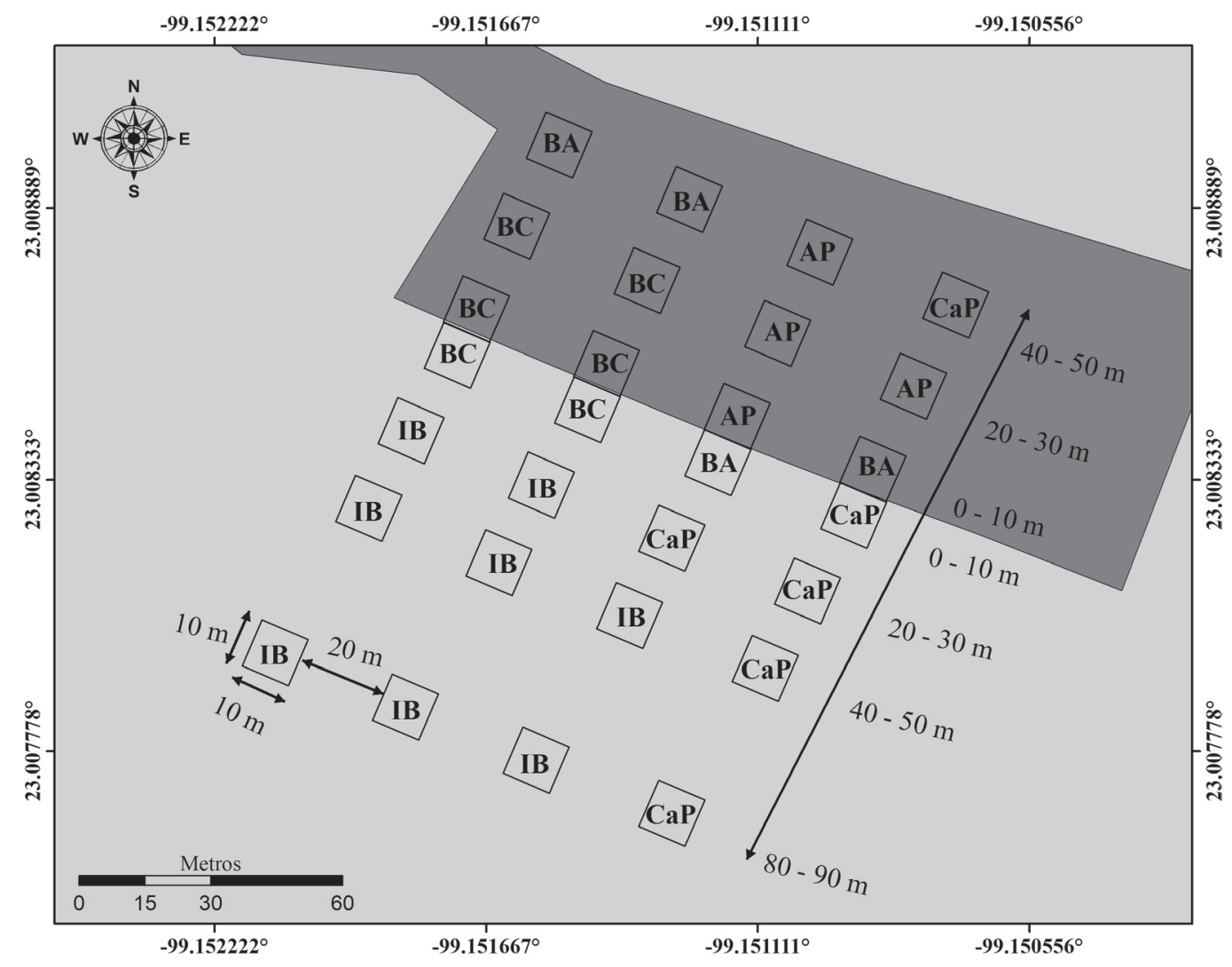

Figura 2. Distribución de las condiciones ambientales dentro del sitio de muestreo, determinadas mediante el análisis de $\mathrm{K}$ medias generalizado. AP: Área perturbada; BA: Borde abierto; BC: Borde cerrado; CaP: Condiciones cercanas al promedio; IB: Interior del bosque. 
$\mathrm{mm} \mathrm{f} / 4 \mathrm{~L}$ ) con $8 \mathrm{~mm}$ de distancia focal colocada a $50 \mathrm{~cm}$ del suelo (Frazer et al. 1999, Asanok et al. 2012). Las imágenes fueron analizadas utilizando el programa Gap Light Analyzer V2 (Frazer et al. 1999) para estimar el porcentaje de apertura del dosel (\% AD), apertura del sitio (\% AS), área foliar efectiva a un zenit de $0-60^{\circ}$ (IAFE60), área foliar efectiva a un zenit de $0-75^{\circ}$ (IAFE75), radiación directa sobre el dosel (RDSD), transmitancia directa bajo el dosel (TDBD) e índice de transmisión de luz ( $\%$ trans dir).

El ambiente termal y de humedad relativa fue caracterizado durante 12 meses (de enero a diciembre de 2016), para ello se colocaron 13 registradores automáticos HOBO (OnSet) separados entre sí por $35 \mathrm{~m}$ (Figura 1) y que cubriera el área conservada y perturbada. Los registradores midieron la temperatura del aire y la humedad relativa cada quince minutos. A partir de los registros obtenidos se estimó el valor máximo, mínimo y promedio, así como el promedio de la diferencia de estas variables entre el día $(3 \mathrm{pm})$ y la noche ( $3 \mathrm{am}$ ) de la temperatura ambiental y la humedad relativa. Los valores de las áreas entre registradores se obtuvieron generando superficies termales y de humedad relativa a partir de la interpolación de datos puntuales con el algoritmo de ponderación de distancia inversa. Los valores de cada cuadrante fueron obtenidos a través de una extracción del centro de cuadrante a partir de la función "extract multiple values" en el software ArcGis 10.2.2 (Childs 2004, Eldrandaly \& Abu-Zaid 2011). Las superficies ambientales y la extracción de los datos fueron realizadas a través del programa ArcGis 10.2.2 (ESRI 2014).

Análisis de datos. Las unidades de muestreo fueron agrupadas en función de sus características ambientales a partir del método divisible de $\mathrm{K}$ medias generalizado. El análisis permite estimar el número de categorías, así como identificar las observaciones que las conforman (Fahmi et al. 2011). De esta manera, se identificaron cinco áreas con características ambientales diferentes (Tabla 1). En el área perturbada y el borde se encontraron tres condiciones ambientales (Figura 2): una categoría denominada "área perturbada", la cual presentó una incidencia lumínica alta, temperaturas altas y humedad relativa baja; el "borde abierto" caracterizado por una baja humedad relativa, así como por presentar una incidencia lumínica y temperaturas cercanas a la media; el "borde cerrado" que se caracterizó por presentar temperaturas altas, así como humedad e incidencia lumínica bajas. En el interior del bosque tropical subcaducifolio conservado se encontraron dos categorías: el "interior cercano al promedio" caracterizado por presentar valores de incidencia lumínica, temperatura y humedad cercanos a la media; y el "interior" el cual a diferencia del "interior cercano al promedio" presentó los valores de humedad relativa más alta.

La relación de los estadios de desarrollo con las características ambientales a lo largo del gradiente se realizó con el índice de marginalidad media (OMI por sus siglas en inglés). El OMI es una técnica multivariada que permite comparar las condiciones ambientales promedio utilizadas por cada estadio (centroide) con respecto a las condiciones promedio del sitio de evaluación (centro de gravedad) (Dolédec et al.
2000). El análisis ordenó los sitios de muestreo en función de las características ambientales con el análisis de componentes principales, la posición canónica de cada uno de los cuadrantes se relacionó con la abundancia de los estadios de desarrollo. El OMI generó un nuevo sistema de referencia (eje) el cual refleja un gradiente ambiental en el que fueron registrados los estadios de desarrollo. Además, en las gráficas obtenidas en el OMI se incorporaron los resultados del análisis de $\mathrm{K}$ medias, con el objetivo de representar las categorías de condiciones ambientales generadas por el efecto borde. El análisis fue realizado utilizando el programa ADE-4 (Thioulouse et al. 1997).

Las características ambientales de los cuadrantes en donde fueron registrados los estadios de desarrollo fueron comparadas con el análisis de funciones discriminantes lineales (AFD). El AFD es una técnica multivariada que incorpora características del análisis de varianza y de los modelos de regresión lineales, que permiten contrastar categorías identificando aquellas características que discriminan las diferencias entre los grupos (Legendre \& Legendre 1998). El análisis fue realizado para cada especie de manera independiente, utilizando como variable independiente las características ambientales registradas en cada muestra (Venegas-Barrera et al. 2015).

Finalmente, se asoció la abundancia de cada estadio de desarrollo y las condiciones ambientales con el análisis de correspondencia simple (AC). El AC es una técnica multivariada que analiza la relación entre más de dos variables categóricas a través de tablas de contingencia (matriz de frecuencias), realizando a su vez una prueba de significancia estadística de $\chi^{2}$ (Härdle \& Simar 2012, Picard et al. 2012). La prueba fue realizada de manera independiente para cada especie, considerando la abundancia por estadio registrada en cada tipo de ambiente resultante del análisis de K-medias. Los análisis de $\mathrm{K}$ medias, AFD y AC fueron realizados a través del programa Statistica 12 (StatSoft Inc. 2014).

\section{Resultados}

Abundancia por estadios de desarrollo. Los adultos juveniles concentraron la mayor abundancia (132 individuos), mientras que el estadio juvenil fue el menos abundante ( 38 individuos), siendo la abundancia en las plántulas y adultos maduros similares (ambos con 94 individuos, Tabla 2). Las especies que presentaron las mayores frecuencias y abundancias fueron Cedrela odorata (113 individuos) y Bursera simaruba (110 individuos), mientras que Guazuma ulmifolia y Lysiloma divaricatum fueron menos abundantes (62 y 73 individuos respectivamente).

Respuesta entre especies. Las especies C. odorata y L. divaricatum mostraron una separación significativa entre su centroide y el centro de gravedad $(P<0.05)$, encontrándose en condiciones diferentes al promedio de todos los cuadrantes (Tabla 3). El centroide de $C$. odorata, se relacionó positivamente a la diferencia de temperatura entre el día y la noche (Figura 3), así como con la radiación directa sobre el dosel $\left(>12.18 \mathrm{~mol} / \mathrm{m}^{2} / \mathrm{d}\right)$ y el porcentaje de apertura del 
Tabla 1. Promedio de los atributos ambientales para cada una de las condiciones ambientales determinadas mediante el análisis de $\mathrm{K}$ medias generalizado.

\begin{tabular}{|c|c|c|c|c|c|}
\hline Atributos ambientales & Área perturbada & Borde abierto & Borde cerrado & Cercano al promedio & Interior \\
\hline Tme $\left({ }^{\circ} \mathrm{C}\right)$ & 21.77 & 21.78 & 22.02 & 21.47 & 21.20 \\
\hline $\operatorname{Tmi}\left({ }^{\circ} \mathrm{C}\right)$ & 9.67 & 9.61 & 9.12 & 9.23 & 8.68 \\
\hline $\operatorname{Tmx}\left({ }^{\circ} \mathrm{C}\right)$ & 37.00 & 37.44 & 39.61 & 36.19 & 34.34 \\
\hline $\operatorname{Tdn}\left({ }^{\circ} \mathrm{C}\right)$ & -8.89 & -8.91 & -11.95 & -9.24 & -10.17 \\
\hline Hme $(\%)$ & 72.83 & 73.22 & 75.53 & 73.95 & 75.60 \\
\hline $\operatorname{Hmi}(\%)$ & 24.19 & 24.07 & 21.56 & 25.94 & 29.00 \\
\hline $\operatorname{Hdn}(\%)$ & -36.04 & -35.61 & -24.60 & -34.96 & -31.76 \\
\hline $\mathrm{AD}(\%)$ & 31.928 & 15.288 & 8.213 & 7.395 & 9.509 \\
\hline AS (\%) & 31.910 & 15.280 & 8.205 & 7.387 & 9.501 \\
\hline IAF4 & 1.335 & 2.498 & 3.550 & 3.745 & 3.454 \\
\hline $\mathrm{IAF}^{\circ}$ & 1.198 & 2.095 & 2.952 & 3.228 & 2.924 \\
\hline $\operatorname{RDSD}\left(\mathrm{mol} / \mathrm{m}^{2} / \mathrm{d}\right)$ & 12.185 & 12.113 & 11.923 & 12.197 & 11.944 \\
\hline $\operatorname{TDBD}\left(\mathrm{mol} / \mathrm{m}^{2} / \mathrm{d}\right)$ & 2.645 & 3.103 & 0.840 & 0.828 & 0.759 \\
\hline ITra (\%) & 21.698 & 25.580 & 7.042 & 6.795 & 6.350 \\
\hline
\end{tabular}

Tme: Temperatura ambiental promedio; Tmi: Temperatura mínima; Tmx: Temperatura máxima; Tdn: Diferencia promedio de la temperatura entre el día y la noche; Hme: Humedad relativa promedio; Hmi: Humedad mínima; Hdn: Diferencia promedio de la humedad entre el día y la noche; AD: Apertura del dosel; IAF4: Índice de área foliar a $60^{\circ}$ de cenit; IAF5: Índice de área foliar a $60^{\circ}$ de cenit; RDSD: Radiación solar directa sobre el dosel; TDBD: Transmitancia directa bajo el dosel; ITra: Relación entre la transmitancia y la radiación directa.

dosel (> $32 \%$ ). En los casos de B. simaruba, G. ulmifolia y L. divaricatum, la abundancia se relacionó positivamente con la temperatura del aire promedio, la temperatura máxima y la transmitancia directa por debajo del dosel (Figura 3).

Variaciones ambientales entre estadios de desarrollo. Los atributos ambientales asociados a los estadios de desarrollo difirieron significativamente en al menos en un estadio en tres especies, siendo L. divaricatum la excepción (Wilks Lambda $=0.65, F_{24,180}=1.19, P=0.25$, Tabla 4). Las características ambientales que aportan la mayor varianza fueron la temperatura del aire promedio, la humedad mínima y la transmitancia directa por debajo del dosel en las tres especies (Figura 4 D-F).

Los cuadrantes donde fueron registrados los adultos de B. simaruba fueron diferentes con respecto al resto de los estadios, y las características de los cuadrantes en donde se encontraron a los adultos juveniles con respecto a los cuadrantes donde fueron registradas las plántulas (Wilks Lambda $=0.43, F_{21,287}=4, P<0.01$, Figura 4A). Los adultos maduros se encontraron en cuadrantes con una temperatura ambiental media de $21.3{ }^{\circ} \mathrm{C}$ y humedad relativa mínima 
Tabla 2. Abundancia registrada por estadio de desarrollo para cada una de las especies analizadas a lo largo del gradiente ambiental (perturbadoborde-conservado) en un fragmento de bosque tropical subcaducifolio en la Reserva de la Biosfera El Cielo.

\begin{tabular}{|c|c|c|c|c|c|c|c|}
\hline \multirow{3}{*}{ Especie } & \multirow{3}{*}{$\begin{array}{l}\text { Estadio de } \\
\text { desarrollo }\end{array}$} & \multicolumn{5}{|c|}{ Gradiente } & \multirow{3}{*}{ Total } \\
\hline & & \multirow[t]{2}{*}{ Perturbado } & \multicolumn{2}{|c|}{ Borde } & \multicolumn{2}{|c|}{ Conservado } & \\
\hline & & & Abierto & Cerrado & $\begin{array}{l}\text { Cercano al } \\
\text { promedio }\end{array}$ & Interior & \\
\hline \multirow{4}{*}{ Bursera simaruba } & Plántulas & 3 & 7 & 8 & 12 & 15 & 45 \\
\hline & Juveniles & 2 & 1 & 4 & 5 & 0 & 12 \\
\hline & $\begin{array}{l}\text { Adultos } \\
\text { juveniles }\end{array}$ & 4 & 10 & 15 & 7 & 0 & 36 \\
\hline & $\begin{array}{l}\text { Adultos } \\
\text { maduros }\end{array}$ & 0 & 0 & 1 & 5 & 11 & 17 \\
\hline \multirow{4}{*}{ Cedrela odorata } & Plántulas & 7 & 7 & 1 & 1 & 0 & 16 \\
\hline & Juveniles & 5 & 1 & 0 & 2 & 1 & 9 \\
\hline & $\begin{array}{l}\text { Adultos } \\
\text { juveniles }\end{array}$ & 20 & 1 & 0 & 6 & 0 & 27 \\
\hline & $\begin{array}{l}\text { Adultos } \\
\text { maduros }\end{array}$ & 29 & 5 & 8 & 19 & 0 & 61 \\
\hline \multirow{4}{*}{ Guazuma ulmifolia } & Plántulas & 1 & 3 & 4 & 8 & 0 & 16 \\
\hline & Juveniles & 2 & 5 & 1 & 2 & 0 & 10 \\
\hline & $\begin{array}{l}\text { Adultos } \\
\text { juveniles }\end{array}$ & 9 & 9 & 5 & 3 & 3 & 29 \\
\hline & $\begin{array}{l}\text { Adultos } \\
\text { maduros }\end{array}$ & 0 & 0 & 1 & 1 & 5 & 7 \\
\hline \multirow{4}{*}{ Lysiloma divaricatum } & Plántulas & 3 & 8 & 2 & 4 & 0 & 17 \\
\hline & Juveniles & 1 & 1 & 1 & 3 & 1 & 7 \\
\hline & $\begin{array}{l}\text { Adultos } \\
\text { juveniles }\end{array}$ & 6 & 16 & 11 & 6 & 1 & 40 \\
\hline & $\begin{array}{l}\text { Adultos } \\
\text { maduros }\end{array}$ & 0 & 4 & 5 & 0 & 0 & 9 \\
\hline
\end{tabular}

de $28.1 \%$ (Tabla 5). En contraste, los adultos juveniles se encontraron en una temperatura promedio mayor y humedad relativa menor. Las plántulas e individuos juveniles mostraron una asociación con la temperatura del aire y humedad cercana al promedio, difiriendo entre sí en la transmitancia por debajo del dosel (plántulas con $1.49 \mathrm{~mol} / \mathrm{m}^{2} / \mathrm{d}$ y juveniles con $1.97 \mathrm{~mol} / \mathrm{m}^{2} / \mathrm{d}$ ).

Las plántulas en $C$. odorata se encontraron en cuadrantes con características ambientales diferentes al estadio juvenil, y los adultos se encontraron en áreas donde la temperatura promedio fue mayor y la humedad relativa menor con respecto a los juveniles y plántulas (Wilks Lambda $=0.45, F_{24,269}=3.84$,
$P<0.01$, Figura 4B). Los cuadrantes donde fueron registradas las plántulas presentaron una mayor transmitancia directa por debajo del dosel $\left(2.83 \mathrm{~mol} / \mathrm{m}^{2} / \mathrm{d}\right)$ con respecto al resto de los estadios $\left(<2.5 \mathrm{~mol} / \mathrm{m}^{2} / \mathrm{d}\right.$, Tabla 5). Finalmente, los adultos maduros fueron registrados en condiciones de mayor temperatura $\left(21.85{ }^{\circ} \mathrm{C}\right)$, mientras que los adultos juveniles requieren de ambientes cercanos al promedio $\left(2.45 \mathrm{~mol} / \mathrm{m}^{2} / \mathrm{d}\right.$ y $\left.21.82{ }^{\circ} \mathrm{C}\right)$ para ambas características ambientales.

Las características ambientales asociadas a los adultos maduros de G. ulmifolia fueron diferentes al resto de los estadios (Wilks Lambda $=0.37, F_{24,128}=2.49, P<0.01$, Figura $4 \mathrm{C}$ ). Los adultos maduros se asociaron a condiciones 
Tabla 3. Resumen de los parámetros del análisis de marginalidad media.

\begin{tabular}{lcccccccc}
\hline Especie & Iner & OMI & T1 & T2 & d2* & T1* & T2* & P \\
\hline Bursera simaruba & 12.8 & 312.7 & 2.96 & 9.80 & 2 & 231 & 766 & 0.94 \\
Cedrela odorata & 19.5 & 6.21 & 4.62 & 8.71 & 318 & 236 & 446 & $<0.01$ \\
Guazuma ulmifolia & 16.2 & 94.36 & 6.63 & 8.62 & 58 & 409 & 532 & 0.07 \\
Lysiloma divaricatum & 15.3 & 1.90 & 3.81 & 9.56 & 124 & 250 & 626 & $<0.01$ \\
\hline
\end{tabular}

Iner: Inercia; OMI: Índice de marginalidad media; T1: Tolerancia; T2: Tolerancia residual; *Proporción aportada a la inercia; $P$ : significancia de la prueba de Monte Carlo.

de menor temperatura ambiental $\left(21.33^{\circ} \mathrm{C}\right)$ y alta humedad (>75\%), mientras que el resto de los estadios se asociaron a condiciones cercanas al promedio. Considerando la transmitancia directa por debajo del dosel, los estadios juveniles $\mathrm{y}$ adultos juveniles se asociaron a condiciones de mayor incidencia $\left(2.8 \mathrm{~mol} / \mathrm{m}^{2} / \mathrm{d}\right.$ y $1.93 \mathrm{~mol} / \mathrm{m}^{2} / \mathrm{d}$ respectivamente), en comparación con las plántulas y los adultos maduros $\left(<1.5 \mathrm{~mol} / \mathrm{m}^{2} / \mathrm{d}\right)$.

Asociación de la abundancia a lo largo del gradiente. Las condiciones ambientales a las cuales se encontraron asociadas las abundancias de cada estadio de desarrollo fueron diferentes en todas las especies (Figura 5). Las plántulas y los juveniles presentaron una mayor ocurrencia en zonas de alta incidencia lumínica ("área perturbada", "bordes abiertos" e "interior cercano al promedio"), mientras que los adultos maduros se asociaron a las condiciones de los "bordes cerrados" y el "interior".

Las plántulas de $B$. simaruba $\left(\chi_{12}^{2}=43.75, P<0.01\right)$ se asociaron a las condiciones del "interior cercano al promedio", mientras que el estadio juvenil se asoció a las condiciones del "área perturbada", los adultos juveniles a "bordes cerrados" y los individuos adultos a las condiciones del "interior" (Figura 5A). Las plántulas de C. odorata se asociaron a las condiciones "borde abierto", el estadio juvenil y adulto maduro a las condiciones de las zonas perturbadas, mientras que el estadio juvenil a las condiciones del "interior cercano al promedio" $\left(\chi_{12}^{2}=37.85, P<0.01\right.$, Figura $\left.5 \mathrm{~B}\right)$. Las plántulas de G. ulmifolia mostraron una asociación con las condiciones del "interior cercano al promedio", el estadio juvenil y adulto juvenil a las condiciones de los "bordes abiertos" mientras que los adultos maduros a las condiciones del "interior" $\left(\chi_{12}^{2}=39.62, P<0.01\right.$, Figura 5C). Finalmente en $L$. divaricatum $\left(\chi_{6}^{2}=13.03, P=0.04\right)$ las plántulas se asocian con las condiciones de los "bordes abiertos", el estadio juvenil al "interior cercano al promedio" y adulto maduro a "bordes cerrados" (Figura 5D).

\section{Discusión}

El presente trabajo muestra que la abundancia al efecto de borde varía entre los estadios de desarrollo debido a que las características ambientales a las que se asocian son diferentes en las especies analizadas. Sin embargo, las plántulas se en-
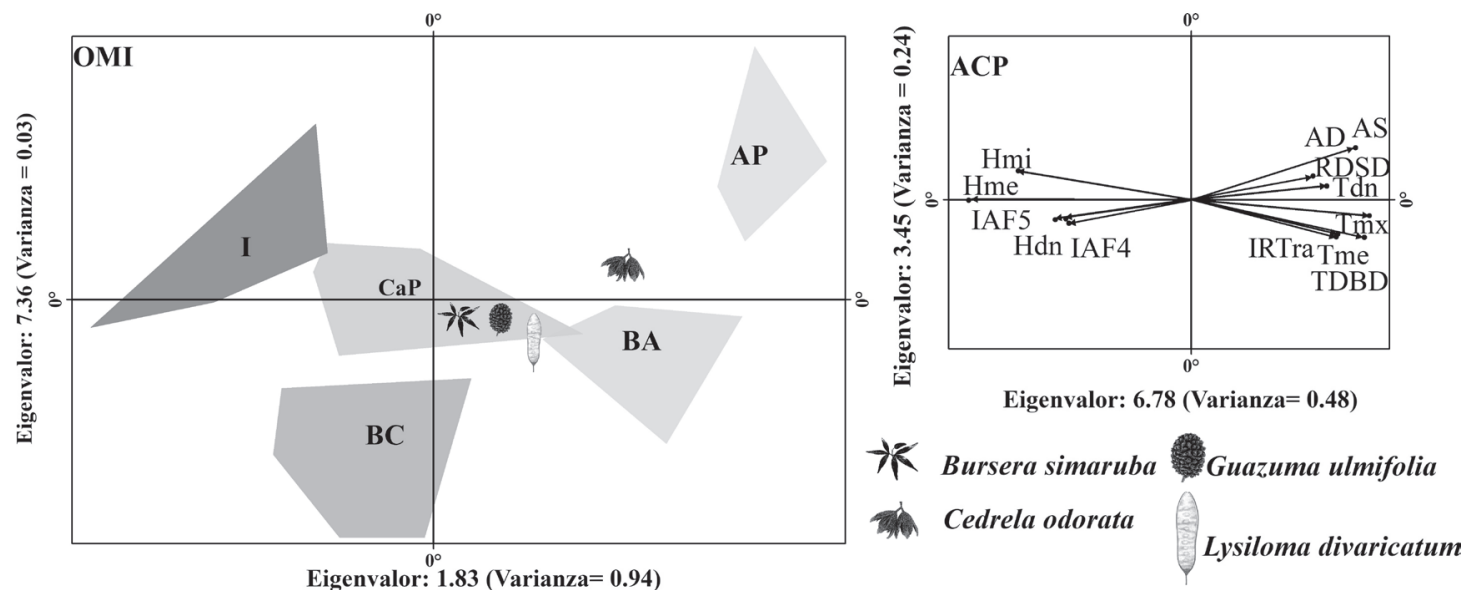

Figura 3. Resultados del análisis de marginalidad media (OMI). Los polígonos en escala de grises representan las unidades de muestreo y las condiciones ambientales obtenidas por el análisis de K medias. AP: Área perturbada; BA: Borde abierto; BC: Borde cerrado; CaP: Cercanas al promedio; I: Interior. Tme: Temperatura media; Tmx: Temperatura máxima; Tmi; Temperatura mínima; Tdn: Diferencia promedio de la temperatura entre el día y la noche; Hme: Humedad relativa promedio; Hmi: Humedad mínima; Hdn: Diferencia promedio de la humedad entre el día y la noche; AD: Apertura del dosel; IAF4: Índice de área foliar a $60^{\circ}$ de cenit; IAF5: Índice de área foliar a $75^{\circ}$ de cenit; RDSD: Radiación solar directa sobre el dosel; TDBD: Transmitancia directa bajo el dosel; IRTra: Relación entre la transmitancia y la radiación directa. 
Tabla 4. Matriz de la prueba de significancia de los análisis de funciones discriminantes de las características ambientales entre los estadios de desarrollo.

\begin{tabular}{llcccc}
\hline Especie & Estadio & Plántula & Juvenil & Adulto juvenil & Adulto maduro \\
\hline \multirow{3}{*}{ B. simaruba } & Plántula & & 0.486 & 0.000 & 0.013 \\
& Juvenil & 1.373 & & 0.012 & 0.046 \\
& Adulto juvenil & 6.955 & 2.867 & 10.476 & 0.000 \\
Adulto maduro & 2.396 & 2.559 & 0.012 & 0.013 \\
C. odorata & Plántula & & 0.486 & & 0.046 \\
& Juvenil & 4.808 & & 1.977 & 0.000 \\
& Adulto juvenil & 6.815 & 1.906 & 0.053 & 0.001 \\
Adulto maduro & 6.823 & 2.637 & 0.076 & 0.002 \\
\hline
\end{tabular}

Diagonales inferiores valores de f; diagonales superiores valores de $\mathrm{p}$; valores de $\mathrm{p}$ con diferencias estadísticamente significativas en fuente roja

contraron en los sitios cercanos al borde, donde la incidencia lumínica fue mayor. Diversos estudios reportan un patrón de respuesta diferente a los resultados expuestos en el presente trabajo, en los cuales la abundancia de los primeros estadios de desarrollo se relaciona negativamente a la distancia al borde (Benitez-Malvido 1998, Benitez-Malvido \& MartínezRamos 2003).
La mayoría de las investigaciones que analizan las respuestas de las especies al efecto de borde utilizaron a la distancia al límite entre las áreas perturbadas y conservadas como la principal variable predictiva (Nava-Cruz et al. 2007, Gonzalez et al. 2010, Granados-Peláez et al. 2014). Además, asumen que las plántulas y el estadio juvenil responden de forma similar a las variaciones en las condiciones ambienta-
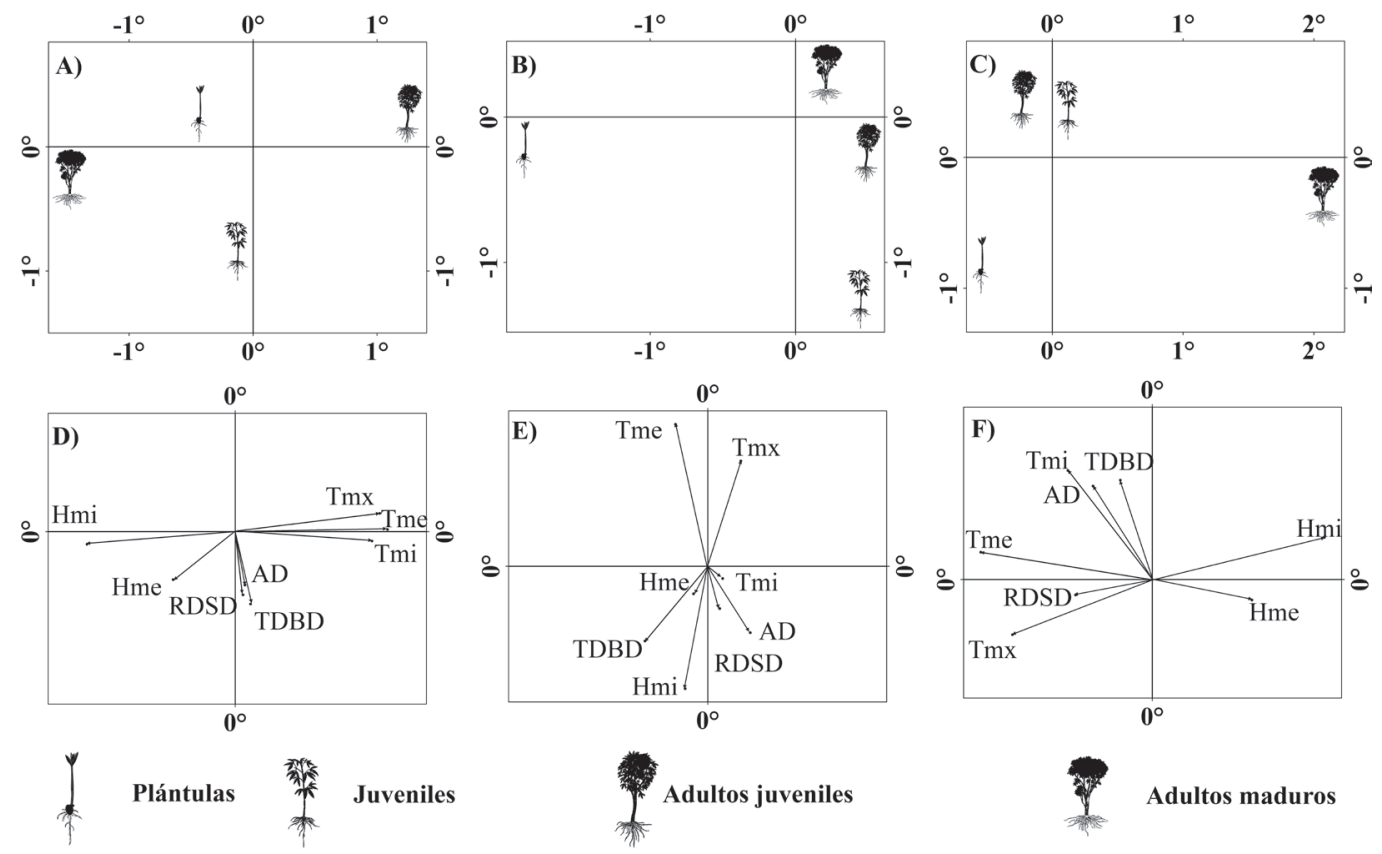

Figura 4. Gráficas de dispersión de los análisis de funciones discriminantes: A-C) Centroides de los estadios de desarrollo; D-F) Estructura de factores; A y D) B. simaruba; B y E) C. odorata; C y F) G. ulmifolia. 
Vargas-Vázquez et al. / Botanical Sciences 97 (1): 35-49. 2019

Tabla 5. Promedio de las características ambientales de cada uno de los estadios de las especies estudiadas.

\begin{tabular}{|c|c|c|c|c|c|c|c|c|c|}
\hline Especie & Estadio & $\begin{array}{l}\text { Tme } \\
\left({ }^{\circ} \mathrm{C}\right) \\
\end{array}$ & $\begin{array}{l}\text { Tmi } \\
\left({ }^{\circ} \mathrm{C}\right) \\
\end{array}$ & $\begin{array}{l}\text { Tmx } \\
\left({ }^{\circ} \mathrm{C}\right) \\
\end{array}$ & $\begin{array}{c}\text { Hme } \\
(\%)\end{array}$ & $\begin{array}{l}\text { Hmi } \\
(\%)\end{array}$ & $\begin{array}{l}\text { AD } \\
(\%)\end{array}$ & $\begin{array}{c}\text { RDSD } \\
\left(\mathrm{mol} / \mathrm{m}^{2} / \mathrm{d}\right)\end{array}$ & $\begin{array}{c}\text { TDBD } \\
\left(\mathrm{mol} / \mathrm{m}^{2} / \mathrm{d}\right)\end{array}$ \\
\hline \multirow{4}{*}{ Bursera simaruba } & Plántula & 21.81 & 9.21 & 38.75 & 73.46 & 22.49 & 12 & 12.11 & 1.44 \\
\hline & Juvenil & 21.76 & 9.54 & 37.4 & 73.55 & 24.24 & 16.22 & 12.1 & 2.8 \\
\hline & Adulto juvenil & 21.79 & 9.35 & 37.74 & 73.53 & 23.66 & 16.65 & 12.08 & 1.93 \\
\hline & Adulto maduro & 21.33 & 8.88 & 35.14 & 75.4 & 27.88 & 7.07 & 11.98 & 1.1 \\
\hline \multirow{4}{*}{ Cedrela odorata } & Plántula & 21.86 & 9.47 & 37.5 & 72.89 & 23.45 & 21.11 & 12.16 & 2.83 \\
\hline & Juvenil & 21.72 & 9.43 & 37.41 & 73.12 & 23.95 & 24.46 & 12.16 & 1.86 \\
\hline & Adulto juvenil & 21.82 & 9.56 & 37.81 & 72.64 & 23.25 & 29.76 & 12.18 & 2.45 \\
\hline & Adulto maduro & 21.85 & 9.46 & 38.36 & 72.84 & 22.59 & 22 & 12.15 & 1.66 \\
\hline \multirow{4}{*}{ Guazuma ulmifolia } & Plántula & 21.56 & 9.1 & 36.4 & 74.29 & 25.71 & 12.85 & 12.11 & 1.49 \\
\hline & Juvenil & 21.64 & 9.18 & 36.7 & 74.51 & 25.22 & 15.24 & 12.10 & 1.97 \\
\hline & Adulto juvenil & 21.91 & 9.41 & 38.75 & 73.96 & 22.52 & 13.2 & 12.08 & 1.58 \\
\hline & Adulto maduro & 21.29 & 8.81 & 34.81 & 75.32 & 28.11 & 11.76 & 11.98 & 1.25 \\
\hline
\end{tabular}

Tme: Temperatura ambiental promedio; Tmi: Temperatura mínima; Tmx: Temperatura máxima; Hme: Humedad relativa promedio; Hmi: Humedad mínima; AD: Apertura del dosel; RDSD: Radiación directa sobre el dosel; TDBD: Transmitancia directa bajo el dosel.

les (Benitez-Malvido 1998, Benitez-Malvido \& Martínez-Ramos 2003). Por lo que tales estudios encontraron una correlación negativa entre la proximidad al borde y la abundancia de las plántulas. En contraste, el presente estudio utilizó a las características ambientales como variables predictivas, manteniendo a las plántulas y los juveniles como categorías independientes. Las características ambientales fueron consideradas como variables predictivas porque son discontinuas a lo largo del borde, determinando de esta manera que las plántulas responden de manera diferencial con respecto a los juveniles al efecto de borde. De esta manera, las plántulas fueron más frecuentes en los cuadrantes cercanos al borde, posiblemente porque las condiciones de luz, temperatura y humedad favorecen a la germinación. Por ejemplo, se ha reportado que varias especies del género Bursera son fotoblásticas y requieren de temperaturas altas entre 25 y 35 ${ }^{\circ} \mathrm{C}$ para germinar (Andrés-Hernández \& Espinosa-Organista 2012), por lo que son consideras heliófitas; particularmente B. simaruba puede germinar y colonizar los claros en las selvas, y ser una buena competidora con las malezas. Por otro lado, L. divaricatum y G. ulmifolia son fotoblásticas neutras, con altos porcentajes de germinación ( $\sim 80 \%)$ bajo un amplio rango de temperaturas $\left(15-40{ }^{\circ} \mathrm{C}\right)$, lo que sugiere cierta tolerancia a los sitios abiertos cercanos al borde (Muñoz et al. 2004, Castro-Marín et al. 2005, Cervantes et al. 2014) en concordancia con su hábitat natural (Cordero et al. 2004). Sin embargo, dichas condiciones son desfavorables para el resto de los estadios en las especies estudiadas, exceptuando C. odorata. Esta última especie es considerada como fotoblástica neutra con un rango óptimo de germinación entre 20- $25^{\circ} \mathrm{C}$, y como una especie pionera demandante de luz que sobrevive tanto en sitios abiertos como cerrados, aunque con mayor incidencia en los primeros (Guariguata 2000). Lo anterior evidencia que existe un incremento en la mortandad en la transición entre estos dos estadios. Tal incremento de la mortandad puede atribuirse a la disminución de la humedad (Nava-Cruz et al. 2007), así como un incremento en la depredación postgerminación generados por el efecto de borde (Athayde \& Morellato 2014).

El sitio de estudio correspondió a un bosque tropical, contiguo a un área perturbada con 20 años de sucesión ecológica. Los gradientes ambientales formados a partir de la interacción de estos tipos de ambientes son clasificados como bordes suaves, caracterizados por presentar una menor variabilidad ambiental que los formados por ambientes contrastantes (Ries et al. 2004). Este tipo de bordes son característicos de sitios en estado de sucesión, los cuales pueden presentarse al interior de bosques no perturbados adyacentes a áreas con 50 años de sucesión (Baker et al. 2014). Dentro de las ventajas de la selección de los bordes suaves como objeto de estudios se encuentra la posibilidad de identificar respuestas tanto en los primeros estadios de desarrollo como en los adultos maduros, lo cual fue corroborado con los resultados del presente trabajo. Además los tipos de ambientes de "borde cerrado" y "cercanos al promedio" se encontraron distribuidos tanto en el interior del área perturbada, como en el interior del bosque no perturbado, los cuales se asociaron con las plántulas de las cuatro especies. Estos resultados pueden indicar que la respuesta de la abundancia encontrada corresponde a la influencia del efecto de borde en estas especies y no a la sucesión ecológica. Por otra parte, si bien la influencia de los árboles adultos maduros sobre las características ambientales ha sido ampliamente detallada en varias investigaciones, caracterizada por la disminución de la temperatura del aire y la incidencia lumínica, así como el incremento en la humedad relativa (Sugiyama \& Peterson 2013, Dovčiak \& Brown 2014, Ouin et al. 2015). Sin embargo, las variaciones ambientales generadas por el efecto de borde pueden conllevar a la mortandad de los estadios adultos, conduciendo a una disminución en su abundancia (Lin et al. 2004). Esto pudiera 

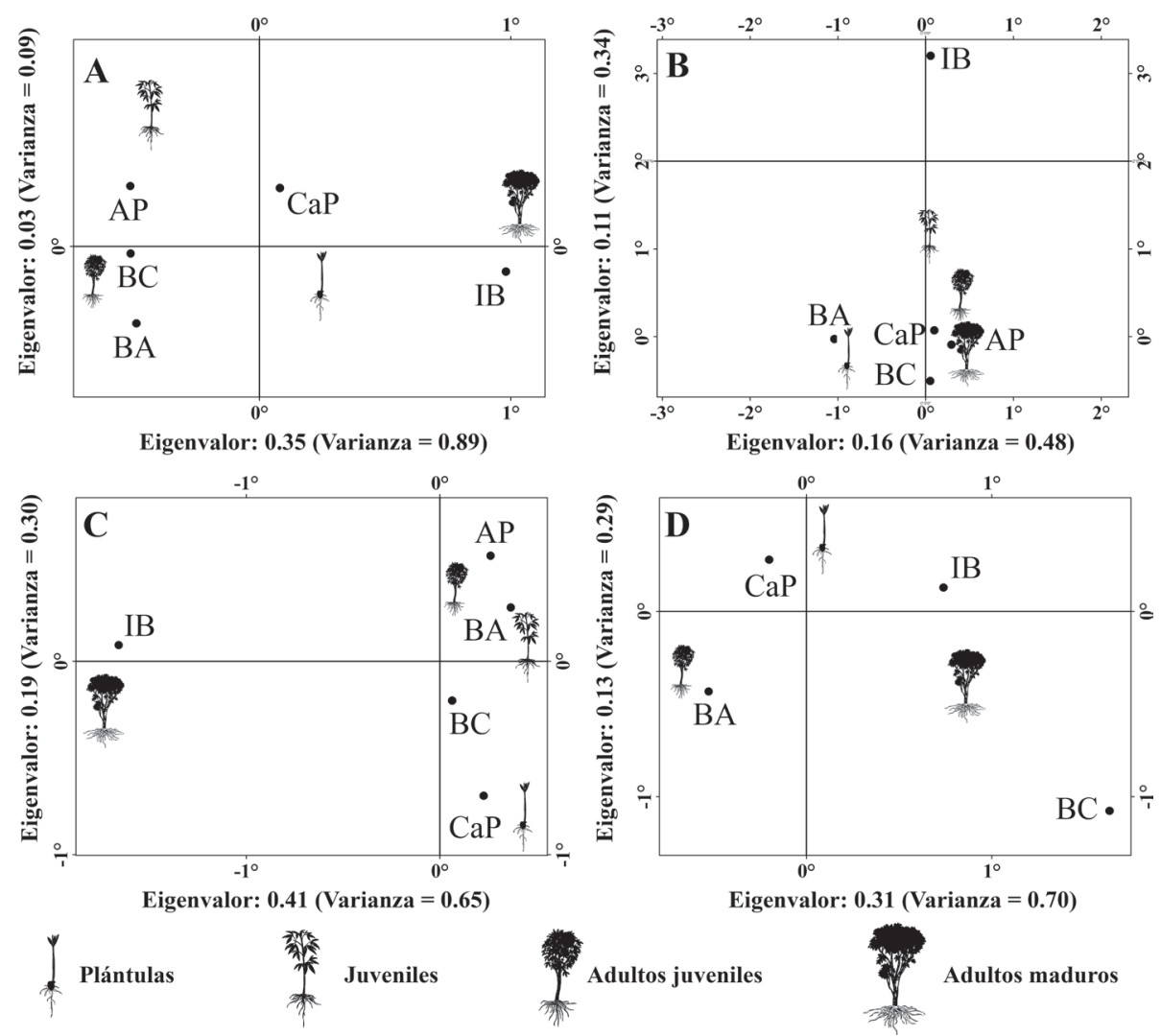

Eigenvalor: $0.31($ Varianza $=\mathbf{0 . 7 0})$

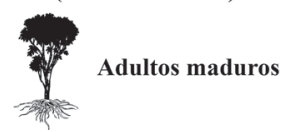

Figura 5. Gráficas de dispersión con la ordenación de los estadios de desarrollo y las condiciones ambientales del análisis de correspondencia. A) B. simaruba; B) C. odorata; C) G. ulmifolia; D) L. divaricatum. AP: Área perturbada; BA: Borde abierto; BC: Borde cerrado; CaP: Cercanas al promedio; I: Interior.

contribuir a la asociación entre el estadio de adultos maduros de B. simaruba, G. ulmifolia y L. divaricatum con las condiciones del interior del bosque.

Los patrones de respuesta de $B$. simaruba, coinciden con los reportados para $B$. fagaroides y B. palmeri (Ortiz-Pulido \& Rico-Gray 2006, Suzán-Azpiri et al. 2017), ya que se encontró asociada a condiciones del interior con una alta incidencia lumínica. Además, las plántulas de $B$. simaruba tienen una mayor probabilidad de encontrarse en bordes abiertos. Esto indica que los ambientes moderadamente sombreados son clave en la germinación de este grupo de especies (Suzán-Azpiri et al. 2017).

La respuesta de $C$. odorata a las variaciones ambientales demuestran que es beneficiada por la presencia de perturbaciones y del efecto borde, lo cual le confiere importancia a esta especie, ya que al tolerar dichas condiciones puede utilizarse en sistemas silvopastoriles para mitigar el efecto borde (Plath et al. 2011). Además, es una de las especies con mayor volumen de madera explotados en México $\left(20,631.88 \mathrm{~m}^{3}\right)$, teniendo un valor estimado de los árboles en pie de MXN $\$ 5,282,183,404$, solo sobrepasado por pinos y encinos (INEGI 2007, Romo-Lozano et al. 2017). La capacidad para incidir en sitios perturbados y bordes en esta especie se debe a que presenta requerimientos altos de incidencia lumínica y tem- peratura, así como de humedad relativa baja. De igual manera, el crecimiento anual de esta especie pudiera ser un factor determinante en el éxito de su supervivencia en estas condiciones, debido a su rápido crecimiento (Dünisch et al. 2003). Sin embargo, la tasa anual de crecimiento no pudo ser considerada en el presente estudio, por lo cual se recomienda que futuros trabajos consideren esta variable para poder explicar el grado en que esta especie responde al efecto de borde.

El tamaño de muestra y el efecto de los afloramientos rocosos sobre la abundancia de las especies son factores que pueden modificar los resultados de la presente investigación. Los afloramientos rocosos incrementan la apertura del dosel, ya que las rocas incrementan la separación entre los árboles. Sin embargo, la selección de especies con alta frecuencia y abundancia aseguró la representatividad del muestreo, lo cual fue confirmado en los análisis. Además, la medición de los atributos ambientales permitió identificar indirectamente los microambientes generados por los afloramientos rocosos, por lo cual no fue necesario su cuantificación.

El presente trabajo plantea un nuevo escenario en el cual es insuficiente sólo analizar las variaciones en la abundancia de las especies, es necesario considerar el efecto de los bordes en cada uno de los estadios de desarrollo. Esto permite identificar los estadios de desarrollo más susceptibles al 
efecto de borde y sobre los cuales es necesario incrementar los esfuerzos de conservación para garantizar la estabilidad de las poblaciones (Williams-Linera \& Lorea 2009). De esta manera, las estrategias de manejo desarrolladas bajo este concepto serían eficaces aún en condiciones de efecto de borde. Además, los atributos ambientales como predictores de las respuestas de las especies deben ser consideradas en el desarrollo de las investigaciones, evitando el uso de la distancia al borde para poder obtener resultados más exactos y extrapolables a cualquier zona geográfica. Además, los atributos ambientales como predictores de las respuestas de la flora local debido a la perturbación y su efecto a áreas conservadas permiten identificar los intervalos ambientales que determinan la abundancia local de las especies.

El presente trabajo presenta la influencia del efecto de borde sobre la abundancia de especies maderables, pero aún es necesario analizar sus efectos sobre la polinización, la producción de semillas y frutos, así como también en la dispersión de las semillas. Tales factores permiten identificar los procesos que conducen a la disminución de la abundancia, así como también estimar con mayor exactitud la dinámica poblacional de los árboles maderables en condiciones de efecto de borde (Wenny 2000, Athayde \& Morellato 2014). De igual manera, la medición de caracteres dendrológicos a diferentes intervalos de tiempo permitiría estimar las implicaciones económicas del efecto de borde sobre el manejo de estas especies de árboles maderables (Binkley et al. 2002, Coomes et al. 2003, Enríquez-Peña \& Suzán-Azpiri 2011). Se espera que estos aspectos sean abordados en futuros trabajos, en los cuales los atributos ambientales sean utilizados como predictores, como lo es el caso de esta investigación.

La disminución en la abundancia del estadio adulto maduro en B. simaruba, G. ulmifolia y L. divaricatum reduce la disponibilidad de ejemplares con las características ideales para su extracción. De esta manera, la extracción de árboles adultos de estas especies en los bordes reduce la disponibilidad de semillas en el suelo, contribuyendo a la disminución de la abundancia de plántulas (Wenny 2000, Sampaio-Diogo et al. 2015). Los resultados encontrados evidencian que los manchones con alta incidencia lumínica, causada por la muerte o la extracción de árboles adultos, favorecen el establecimiento de las plántulas pero son desfavorables para el estadio juvenil (Renaud et al. 2011, Tanga et al. 2014). De esta manera, la disponibilidad de ejemplares aprovechables dentro del borde en un periodo mayor de tiempo pudiera disminuir, imposibilitando su manejo (Athayde \& Morellato 2014). Por ello, es necesario reducir la pérdida de cobertura de los bosques tropicales y tomar medidas para mitigar el efecto de borde para poder garantizar la disponibilidad de individuos aprovechables de estas especies.

\section{Agradecimientos}

Este estudio fue financiado por el proyecto de Ciencia Básica SEP-CONACYT: 0219583. El primer autor también agradece al CONACYT por la beca de manutención (397894) percibida durante su estudio de Doctorado en Ecología y Manejo de Recursos Naturales.

\section{Literatura citada}

Álvarez-Buylla ER, Martínez-Ramos M. 1992. Demography and allometry of Cecropia obtusifolia, a neotropical pioneer tree - An evaluation of the climax-pioneer paradigm for tropical rain forests. Journal of Ecology 80: 275-290.

DOI: http://doi.org/10.2307/2261011

Andrés-Hernández AR, Espinosa-Organista D. 2002. Morfología de plantulas de Bursera Jacq. ex L. y sus implicaciones filogenéticas. Boletín de la Sociedad Botánica de México 70: 5-12. DOI: http://doi.org/10.17129/botsci.1652

Asanok L, Marod D, Pattanavibool A, Nakashizuka T. 2012. Colonization of tree species along an interior-exterior gradient across the forest edge in a tropical montane forest, northwest Thailand. Tropics 21: 67-80.

DOI: http://doi.org/10.3759/tropics.21.67

Athayde EA, Morellato LPC. 2014. Anthropogenic edges, isolation and the flowering time and fruit set of Anadenanthera peregrina, a cerrado savanna tree. International Journal of Biometeorology 58: 443-454.

DOI: http://doi.org/10.1007/s00484-013-0727-y

Baker TP, Jordan GJ, Steel EA, Fountain-Jones NM, Wardlaw TJ, Baker SC. 2014. Microclimate through space and time: Microclimatic variation at the edge of regeneration forests over daily, yearly and decadal time scales. Forest Ecology and Management 334: 174-184.

DOI: http://doi.org/10.1016/j.foreco.2014.09.008

Bárcenas-Pazos GM. 1995. Caracterización tecnológica de veinte especies maderables de la selva Lacandona, Chis., México. Madera y Bosques 1: 9-38.

Barrera-Salazar A, Mandujano S, Villarreal-Espino-Barros OA, Jiménez-García D. 2015. Classification of vegetation types in the habitat of white tailed deer in a location of the TehuacánCuicatlán Biosphere Reserve, Mexico. Tropical Conservation Science 8: 547-563.

DOI: http://doi.org/10.1177/194008291500800217

Benítez-Malvido J. 1998. Impact of forest fragmentation on seedling abundance in a tropical rain forest. Conservation Biology 12: 380-389.

DOI: http://doi.org/10.1111/j.1523-1739.1998.96295.x

Benítez-Malvido J, Martínez-Ramos M. 2003. Influence of edge exposure on tree seedling species recruitment in tropical rain forest Fragments. Biotropica 35: 530-541.

DOI: http://doi.org/10.1111/j.1744-7429.2003.tb00609.x

Binkley D, Stape LJ, Ryan GM, Barnard RH, Fownes J. 2002. Age-related decline in forest ecosystem growth: An individual-tree, stand-structure hypothesis. Ecosystems 5: 58-67. DOI: http://doi.org/10.1007/s10021-001-0055-7

Castro-Marín G, Nygård R, González-Rivas B, Odén PC. 2005. Stand dynamics and basal area change in atropical dry forest reserve in Nicaragua. Forest Ecology \& Management 208: 63-75. DOI: http://doi.org/10.1016/j.foreco.2004.10.072

Cervantes M, Ceccon E, Bonfil C. 2014. Germination of stored seeds of four tree species from the tropical dry forest of Morelos, Mexico. Botanical Sciences 92: 281-287. DOI: http://doi.org/10.17129/botsci.96

Clark Labs. 2014. IDRISI Selva: versión 17.0. Worcester, Massachusetts. Clark University. $<$ https://clarklabs.org/do- 
wnload/idrisi-selva-service-update-to-17-2/> (accessed: 4/10/2015)

CONABIO [Comisión Nacional para el Conocimiento y Uso de la Biodiversidad]. 1998. Climas (Clasificación de Koppen, modificado por García). Escala 1:1000000. México, D.F. $<$ http:/www.CONABIO.gob.mx/informacion/gis/> (accessed: $1 / 1 / 2017)$

CONABIO. 2015. Áreas Naturales Protegidas Estatales, Municipales, Ejidales y Privadas de México 2015. 1: 250,000. México, DF. <http://www.CONABIO.gob.mx/informacion/ gis/> (accessed: 22/10/15)

Coomes DA, Duncan RP, Allen RB, Truscott J. 2003. Disturbances prevent stem size-density distributions in natural forests from following scaling relationships. Ecology Letters 6: 980989. DOI: http://doi.org/10.1046/j.1461-0248.2003.00520.x

Cordero J, Mesén F, Montero M, Stewart J, Boshier D, Chamberlain J, Pennington T, Hands M, Hughes C, Detlefsen G. 2004. Descripción de especies de árboles nativos de América Central. In: Cordero J, Boshier D, eds. Manual de Arboles de Centroamérica. Centro Agronómico de Investigación y Enseñanza. 313-958. ISBN: 9780850741612

Challenger A, Dirzo R, López-Acosta JC, Mendoza E, LiraNoriega A, Cruz I. 2009. Factores de cambio y estado de la biodiversidad. In: CONABIO ed._Capital natural de México. Comisión Nacional para el Conocimiento y Uso de la Biodiversidad, México. 37-73. ISBN: 978-607-7607 03-8.

Cheesman AW, Winter K. 2013. Elevated night-time temperatures increase growth in seedlings of two tropical pioneer tree species. New Phytologist 197: 1185-1192.

DOI: http://doi.org/10.1111/nph.12098

Childs C. 2004. Interpolating surfaces in ArcGIS spatial analyst. ArcUser 2004: 32-35.

Dolédec S, Chessel D, Gimaret-Carpentier C. 2000. Niche separation in community analysis: a new method. Ecology 81: 2914-2927. DOI:

http://doi.org/10.1890/0012-9658(2000)081[2914: NSICAA]2.0.CO;2

Dovčiak M, Brown J. 2014. Secondary edge effects in regenerating forest landscapes: vegetation and microclimate patterns and their implications for management and conservation. New Forests 45: 733-744.

DOI: http://doi.org/10.1007/s11056-014-9419-7

Dünisch O, Montóia VR, Bauch J. 2003. Dendroecological investigations on Swietenia macrophylla King and Cedrela odorata L. (Meliaceae) in the central Amazon. Trees 17: 244-250. DOI: http://doi.org/10.1007/s00468-002-0230-2

Eldrandaly KA, Abu-Zaid MS. 2011. Comparison of Six GISBased Spatial Interpolation Methods for Estimating Air Temperature in Western Saudi Arabia. Journal of Environmental Informatics 18: 38-45.

DOI: http://doi.org/10.3808/jei.201100197

Enríquez-Peña EG, Suzán-Azpiri H. 2011. Estructura poblacional de Taxodium mucronatum en condiciones contrastantes de perturbación en el estado de Querétaro, México. Revista Mexicana de Biodiversidad 82: 153-167. DOI: http://doi.org/10.22201/ib.20078706e.2011.1.380

ESRI. 2014. ArcGIS Desktop: versión 10.2.2. Redlands, California. Environmental Systems Research Institute. <http:// desktop.arcgis.com/es/arcmap/latest/get-started/setup/arcgisdesktop-quick-start-guide.htm> (accessed: 4/10/2015)

Ewers RM, Banks-Leite C. 2013. Fragmentation impairs the microclimate buffering effect of tropical forests. PLOS ONE 8: e58093. DOI http://doi.org/10.1371/journal.pone.0058093

Fahmi FM, Kartal E, İyigün C, Yozgatligil C, Purutcuoğlu V, Batmaz İ, Türkeş M, Köksal G. 2011. Determining the climate zones of Turkey by center-based clustering methods. In: Nonlinear and Complex Dynamics: Applications in Physical, Biological, and Financial Systems. Baleanu D, Tenreiro-Machado JA, Luo AC. ed. New York: Springer New York. 171-177. ISBN: 978-1-4614-0231-2

Figueroa JA, Lusk CH. 2001. Germination requirements and seedling shade tolerance are not correlated in a Chilean temperate rain forest. New Phytologist 152: 483-489. DOI: http://doi.org/10.1046/j.0028-646X.2001.00282.x

Frazer GW, Canham C, Lertzman K. 1999. Gap Light Analyzer (GLA), Version 2.0: Imaging Software to Extract Canopy Structure and Gap Light Transmission Indices from Truecolour Fisheye Photographs, Users Manual and Program Documentation. Victoria, Canada: Simon Fraser University, Burnaby, British Columbia, and the Institute of Ecosystem Studies, Millbrook, New York.

Fuentes-Salinas M, Correa-Méndez F, Borja-De la Rosa A, Corona-Ambriz A. 2008. Características tecnológicas de 16 maderas del estado de Tamaulipas, que influyen en la fabricación de tableros de partículas y de fibras. Revista Chapingo Serie Ciencias Forestales y del Ambiente 14: 65-71.

González M, Ladet S, Deconchat M, Cabanettes A, Alard D, Balent G. 2010. Relative contribution of edge and interior zones to patch size effect on species richness: An example for woody plants. Forest Ecology and Management 259: 266274. DOI: http://doi.org/10.1016/j.foreco.2009.10.010

Granados-Peláez C, Serrano-Giné D, García-Romero A. 2014. Efecto de borde en la composición y en la estructura de los bosques templados, Sierra de Monte-Alto, Centro de México. Caldasia 36: 269-287.

Guariguata MR. 2000. Seed and seedling ecology of tree species in Neotropical secondary forest: Management Implications. Ecological Applications 10:145-154. DOI: http://doi.org/10.1890/1051-0761(2000)010[0145: SASEOT]2.0.CO;2

Härdle WK, Simar L. 2012. Correspondence analysis. In: Applied Multivariate Statistical Analysis. Berlin: Springer Berlin Heidelberg. 367-384. ISBN-13: 978-3642172281

Harper KA, Macdonald SE. 2002. Structure and composition of edges next to regenerating clear-cuts in mixed-wood boreal forest. Journal of Vegetation Science 13: 535-546. DOI: http://doi.org/10.1111/j.1654-1103.2002.tb02080.x

Harper KA, Macdonald SE, Burton PJ, Chen J, Brosofske KD, Saunders SC, Euskirchen ES, Roberts DAR, Jaiteh MS, Esseen P-A. 2005. Edge influence on forest structure and composition in fragmented landscapes. Conservation Biology 19: 768-782.

DOI: http://doi.org/10.1111/j.1523-1739.2005.00045.x

Hernández-Sandoval L, González-Romo CE, González-Medrano F. 1991. Plantas útiles de Tamaulipas. Anales del Instituto de Biología. Serie Botánica 62: 1-38. 
Herrera R, Ulloa D, Valdés-Lafont O, Priego A, Valdés A. 1997. Ecotechnologies for the sustainable management of tropical diversity. Nature \& Resources 33:2-17.

INEGI [Instituto Nacional de Estadística y Geografía]. 2006. División Municipal de México, 2005. 1:250,000. México, DF. $<$ http://www.CONABIO.gob.mx/informacion/gis/> (accessed 1/1/2017)

INEGI. 2007. Censo Agrícola, Ganadero y Forestal 2007. INEGI. México. <http://www.INEGI.org.mx/est/contenidos/ proyectos/Agro/ca2007/Resultados_Agricola/default.aspx.> (accessed 3/5/2018)

INEGI. 2013. Continuo de Elevaciones Mexicano 3.0 (CEM 3.0). 1:20,000. México, DF. <http://www.INEGI.org.mx/ geo/contenidos/datosrelieve/continental/descarga.aspx $>$ (accessed 22/10/2015)

INIFAP [Instituto Nacional de Investigaciones Forestales, Agrícolas y Pecuarias], CONABIO. 1995. Edafología. Escalas 1:250000. México, D.F. <http://www.CONABIO.gob.mx/ informacion/gis/> (accessed 1/1/2017)

Legendre P, Legendre L. 1998. Numerical Ecology. Amsterdam: Elsevier. eBook ISBN: 9780080523170

Lillesand T, Kiefer RW, Chipman J. 2014. Remote Sensing and Image Interpretation. USA: John Wiley \& Sons. ISBN: 9781-118-34328-9

Lin Y, Hulting ML, Augspurger CK. 2004. Causes of spatial patterns of dead trees in forest fragments in Illinois. Plant Ecology 170: 15-27.

DOI: http://doi.org/10.1023/B:VEGE.0000019017.41546.eb

López-Barrera F. 2004. Estructura y función en bordes de bosques. Ecosistemas 13: 67-77.

López-Barrera F, Newton A. 2005. Edge type effect on germination of oak tree species in the Highlands of Chiapas, Mexico. Forest Ecology and Management 217: 67-79.

DOI: http://doi.org/10.1016/j.foreco.2005.05.048

Lusk CH, Chazdon RL, Hofmann G. 2006. A bounded null model explains juvenile tree community structure along light availability gradients in a temperate rain forest. Oikos 112: 131137. DOI: http://doi.org/10.1111/j.0030-1299.2006.13922.x

Montgomery R, Chazdon R. 2002. Light gradient partitioning by tropical tree seedlings in the absence of canopy gaps. Oecologia 131: 165-174.

DOI: http://doi.org/10.1007/s00442-002-0872-1

Moreno-Casasola P, Paradowska K. 2009. Especies útiles de la selva baja caducifolia en las dunas costeras del centro de Veracruz. Madera y Bosques 15: 21-44.

Muñoz BC, Sánchez JA, Almaguer W. 2004. Germinación, dormancia y longevidad potencial de las semillas de Guazuma ulmifolia. Pastos y Forrajes 27: 25-33.

Murcia C. 1995. Edge effects in fragmented forests: implications for conservation. Trends in Ecology \& Evolution 10: 58-62. DOI: http://doi.org/10.1016/S0169-5347(00)88977-6

Nava-Cruz Y, Maass-Moreno M, Briones-Villareal O, MéndezRamírez I. 2007. Evaluación del efecto borde sobre dos especies del bosque tropical caducifolio de Jalisco, México. Agrociencia 41: 111-120.

Nava-Sosa IJ, Lindig-Cisneros R, Del-Val E, Lara-Cabrera SI. 2010. Limitations for seedling establishment in volcanic tephra deposits. Botanical Sciences 87: 51-59.
DOI: http://doi.org/10.17129/botsci.297

Ouin A, Cabanettes A, Andrieu E, Deconchat M, Roume A, Vigan M, Larrieu L. 2015. Comparison of tree microhabitat abundance and diversity in the edges and interior of small temperate woodlands. Forest Ecology and Management 340: 31-39. DOI: http://doi.org/10.1016/j.foreco.2014.12.009

Ortiz-Pulido R, Rico-Gray V. 2006. Seed dispersal of Bursera fagaroides (Burseraceae): The effect of linking environmental factors. The Southwestern Naturalist 51: 11-21.

Pearson TRH, Burslem DFR, Mullins CE, Dalling JW. 2002. Germination ecology of Neotropical Pioneers: interacting effects of environmental conditions and seed size. Ecology 83: 2798-2807. DOI:

http://doi.org/10.1890/0012-9658(2002)083[2798: GEONPI]2.0.CO;2

Pearson TRH, Burslem DFR, Mullins CE, Dalling JW. 2003. Functional significance of photoblastic germination in Neotropical pioneer trees: a seed's eye view. Functional Ecology 17: 394-402.

DOI: http://doi.org/10.1046/j.1365-2435.2003.00747.x

Picard N, Köhler P, Mortier F, Gourlet-Fleury S. 2012. A comparison of five classifications of species into functional groups in tropical forests of French Guiana. Ecological Complexity 11: 75-83. DOI: http://doi.org/10.1016/j.ecocom.2012.03.003

Plath M, Mody K, Potvin C, Dorn S. 2011. Do multipurpose companion trees affect high value timber trees in a silvopastoral plantation system? Agroforestry Systems 81: 79-92. DOI: http://doi.org/10.1007/s10457-010-9308-9

Poorter L, Arets EJMM. 2003. Light environment and tree strategies in a Bolivian tropical moist forest: an evaluation of the light partitioning hypothesis. Plant Ecology 166: 295-306. DOI: http://doi.org/10.1023/a:1023295806147

Renaud V, Innes JL, Dobbertin M, Rebetez M. 2011. Comparison between open-site and below-canopy climatic conditions in Switzerland for different types of forests over 10 years (1998-2007). Theoretical and Applied Climatology 105: 119-127. DOI: http://doi.org/10.1007/s00704-010-0361-0

Ries L, Fletcher RJ, Battin J, Sisk TD. 2004. Ecological responses to habitat edges: mechanisms, models, and variability explained. Annual Review of Ecology, Evolution, and Systematics. 35:491-522. DOI:

http://doi.org/10.1146/annurev.ecolsys.35.112202.130148

Romo-Lozano JL, Vargas-Hernández JJ, López-Upton J, Ávila Angulo ML. 2017. Estimación del valor financiero de las existencias maderables de cedro rojo (Cedrela odorata L.) en México. Madera y Bosques 23: 111-120.

Rüger N, Berger U, Hubbell SP, Vieilledent G, Condit R. 2011. Growth strategies of tropical tree species: Disentangling light and size effects. PLOS ONE 6: e25330.

DOI: http://doi.org/10.1371/journal.pone.0025330

Salas JB. 1993. Arboles de Nicaragua. Managua: Instituto Nicaragüense de Recursos Naturales y del Ambiente. Servicio Forestal Nacional.

Sampaio-Diogo IJ, Fortunato MEM, Costa IR. 2015. Seed deposition in the edge-interior gradient of a degraded fragment of tropical semideciduous forest, Northeastern Brazil. Revista de Biología Tropical 63: 14.

DOI: http://doi.org/10.15517/rbt.v63i4.17077 
Sánchez-Ramos G, Reyes-Castillo P, Dirzo R. 2005. Historia natural de la Reserva de la Biosfera El Cielo, Tamaulipas, México. Universidad Autónoma de Tamaulipas. ISBN 9687662-67-0

Sapijanskas J, Paquette A, Potvin C, Kunert N, Loreau M. 2014. Tropical tree diversity enhances light capture through crown plasticity and spatial and temporal niche differences. Ecology 95: 2479-2492. DOI: http://doi.org/10.1890/13-1366.1

Silva-Magnago LF, Ferreira-Rocha M, Meyer L, Martins SV, Meira-Neto JAA. 2015. Microclimatic conditions at forest edges have significant impacts on vegetation structure in large Atlantic forest fragments. Biodiversity and Conservation 24: 2305-2318.

DOI: http://doi.org/10.1007/s10531-015-0961-1

StatSoft Inc. 2014. Statistica (Data analysis software system): versión 12. USA: StatSoft Inc.

Steinberg M, Taylor M, Kinney K. 2014. The El Cielo Biosphere Reserve: forest cover changes and conservation attitudes in an important neotropical region. The Professional Geographer 66: 403-411.

DOI: http://doi.org/10.1080/00330124.2013.799994

Sugiyama A, Peterson CJ. 2013. Edge effects act differentially on multiple early regeneration stages of a shade-tolerant tree Tapirira mexicana. Biotropica 45: 37-44.

DOI: http://doi.org/10.1111/j.1744-7429.2012.00897.x

Suzán-Azpiri H, Ponce-González OO, Malda-Barrera GX, Cambrón-Sandoval VH, Carrillo-Ángeles IG. 2017. Edge effect on the population structure and the reproductive success of two Bursera species. Botanical Sciences 95: 9-22. DOI: http://doi.org/10.17129/botsci.775

Tanga AA, Erenso TF, Lemma B. 2014. Effects of three tree species on microclimate and soil amelioration in the central rift valley of Ethiopia. Journal of Soil Science and Environmental Management 5: 62-71.

DOI: http://doi.org/10.5897/JSSEM12.060

Teketay D, Granström A. 1997. Germination ecology of forest

Editor de sección: Alejandro Zavala Hustado

Contribuciones de los autores: VAVV: realizó los experimentos, analizó los datos, escribió el artículo. CSVB: concibió y diseñó los experimentos, escribió el artículo. AMO: diseñó los experimentos, revisó los borradores del documento. JGMÁ: diseñó los experimentos, revisó los borradores del documento. EAR: diseñó los experimentos, revisó los borradores del documento. ERM: revisó los análisis e hizo la edición final del manuscrito. species from the highlands of Ethiopia. Journal of Tropical Ecology 13: 805-831.

DOI: http://doi.org/10.1017/S0266467400011019

Thier O, Wesenberg J. 2016. Floristic composition and edge-induced homogenization in tree communities in the fragmented Atlantic rainforest of Rio de Janeiro, Brazil. Tropical Conservation Science 9: 852-876.

DOI: http://doi.org/10.1177/194008291600900217

Thioulouse J, Chessel D, Dolédec S, Olivier JM. 1997. ADE-4: a multivariate analysis and graphical display software. Statistics and Computing 7: 75-83.

DOI: http://doi.org/10.1023/A:1018513530268

Valiente-Banuet A, González-Medrano F, Piñero-Dalmau D. 1995. La vegetación selvática de la región de Gómez Farías, Tamaulipas, México. Acta Botanica Mexicana 33: 1-36.

Venegas-Barrera CS, Correa-Sandoval A, Mora-Olivo A, HortaVega JV. 2015. Representatividad geográfica y ambiental de gastrópodos, pteridofitas y plantas acuáticas en el estado de Tamaulipas, México. Revista Mexicana de Biodiversidad 86: 719-729. DOI: http://doi.org/10.1016/j.rmb.2015.06.002

Way DA, Oren R. 2010. Differential responses to changes in growth temperature between trees from different functional groups and biomes: a review and synthesis of data Tree Physiology 30: 669-688.

DOI: http://doi.org/10.1093/treephys/tpq015

Wenny DG. 2000. Seed dispersal, seed predation, and seedling recruitment of a Neotropical montane tree. Ecological Monographs 70: 331-351.

DOI: http://doi.org/10.1890/0012-9615(2000)070[0331: SDSPAS]2.0.CO;2

Williams-Linera G, Lorea F. 2009. Tree species diversity driven by environmental and anthropogenic factors in tropical dry forest fragments of central Veracruz, Mexico. Biodiversity and Conservation 18: 3269-3293.

DOI: http://doi.org/10.1007/s10531-009-9641-3 